\title{
Dynamic Intraventricular Obstruction in Acute Myocardial Infarction With Administration of Cilostazol
}

\author{
Kazuyuki Ozaki, MD; Chiyo Maeda, MD; Tsugumi Takayama, MD; Makoto Hoyano, MD; \\ Takao Yanagawa, MD; Keiichi Tsuchida, MD; Kazuyoshi Takahashi, MD; \\ Tsutomu Miida, MD; Hirotaka Oda, MD
}

\begin{abstract}
Dynamic intraventricular obstruction is a less well-known mechanical complication of acute myocardial infarction (AMI). Its hallmark is the development of a new systolic murmur, and echocardiography is necessary for diagnosis. We describe a case of a 74-year-old woman with dynamic intraventricular obstruction complicating AMI. Serial echocardiography suggested that the intraventricular gradient was a consequence of basal hyperkinesis, which was a reciprocal response to akinesis of the apical wall. Cilostazol, which was administered to prevent subacute stent thrombosis after percutaneous coronary intervention, might have contributed to the transient intraventricular obstruction. (Circ J 2007; 71: 608-612)
\end{abstract}

Key Words: Acute myocardial infarction; Cilostazol; Dynamic intraventricular obstruction; Sigmoid septum

$\mathbf{I}$ n cases of acute myocardial infarction (AMI), the acute onset of a systolic murmur sometimes means serious complications, such as mitral regurgitation and ventricular septal perforation, which are usually associated with rapid hemodynamic instability, cardiogenic shock, and ultimately a fatal outcome. Therefore, examination to determine the cause of the systolic murmur is extremely important and urgent. Recently, there have been some reports of dynamic intraventricular obstruction in the setting of AMI that resulted in a systolic murmur ${ }^{1-7}$ Moreover, dynamic intraventricular obstruction could be associated with a catastrophic medical outcome. We report a case of transient dynamic intraventricular obstruction in AMI following administration of cilostazol.

\section{Case Report}

A 74-year-old woman with hypertension and hyperlipidemia was admitted to our hospital for chest pain. Her blood pressure was $124 / 90 \mathrm{mmHg}$ and her heart rate was 75 beats/min. A chest examination revealed no murmur. An electrocardiogram revealed sinus rhythm ( 75 beats $/ \mathrm{min}$ ) and ST elevation in the precordial leads (Fig 1B). She also exhibited an elevated white blood cell count of $11,300 / \mathrm{mm}^{3}$ and a positive heart-type fatty acid binding protein test. A coronary angiography (CAG) was immediately performed and revealed 1-vessel coronary heart disease with total occlusion of the mid-left anterior descending artery (LAD) (Fig 2 Left). A direct percutaneous coronary intervention (PCI) for the occluded LAD was performed. Two metal stents were implanted into the occluded lesion and recanalization was achieved (Fig 2 Right). However, only a minor dissection occurred in the distal edge of the implanted stents.

(Received August 2, 2006; revised manuscript received December 11, 2006; accepted January 16, 2007)

Department of Cardiology, Niigata City General Hospital, Niigata, Japan

Mailing address: Kazuyuki Ozaki, MD, Department of Cardiology, Niigata City General Hospital, 2-6-1 Shichikuyama, Niigata 950-8739,

Japan. E-mail: k-ozaki@hosp.niigata.niigata.jp
Left ventriculography (LVG) demonstrated apical akinesia and posterobasal and posterolateral hypercontractility (Fig 3). Mitral regurgitation was not evident. There was no pressure gradient between the apex and ascending aorta on pullback. After the PCI, the patient was treated with cilostazol (200 mg/day) in addition to aspirin ( $200 \mathrm{mg} /$ day) and ticlopidine ( $200 \mathrm{mg}$ /day) to prevent subacute stent thrombosis. Intravenous administration of isosorbide dinitrate was started immediately after diagnosis of AMI and was continued for $24 \mathrm{~h}$. The patient had taken candesartan cilexetil ( $8 \mathrm{mg}$ /day) for hypertension and it was continued during her stay in hospital. Her peak creatine kinase level was 1,775 IU/L.

On day 4 of the patient's hospitalization, a physical examination revealed a heart rate of 110 beats/min and a blood pressure of $102 / 80 \mathrm{mmHg}$. A grade $3 / 6$ ejection systolic murmur that had not been previously noted was detected at the apex area. A transthoracic echocardiogram showed a sigmoid septum with basal inferolateral hypercontractility and apical akinesis (Fig 4A). There were no findings of left ventricular hypertrophy or asymmetric septal hypertrophy. There were also no indications of mitral regurgitation or systolic anterior motion of the mitral valve leaflets. This examination also demonstrated a peak Doppler velocity of $6.35 \mathrm{~m} / \mathrm{s}$ corresponding to a peak gradient of $161 \mathrm{mmHg}$ across the left ventricular outflow tract (LVOT) (Fig 4B). Consequently, the administration of cilostazol was terminated. During the next day, the peak gradient across LVOT decreased to $111 \mathrm{mmHg}$. On the second day after cessation of cilostazol, the patient's heart rate was 82 beats/min and her blood pressure was $108 / 74 \mathrm{mmHg}$. The systolic ejection murmur was diminished and an echocardiography revealed a complete resolution of the intraventricular obstruction (Fig 4C).

After cardiac rehabilitation was complete, a provocation test with cilostazol ( $200 \mathrm{mg} /$ day $)$ and an exercise stress test were performed. In both tests, neither ejection systolic murmur nor intraventricular obstruction was noted. On hospitalization day 24 , a cardiac catheterization was performed. CAG revealed a patent flow of the treated LAD. LVG 
A

I

II

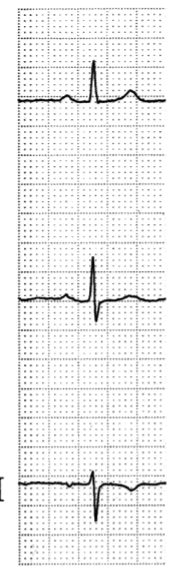

B

1

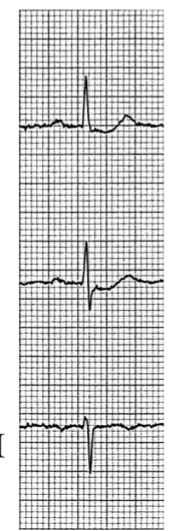

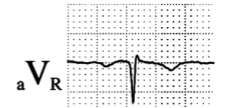
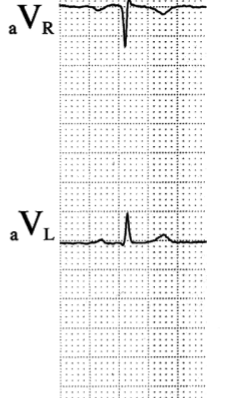

$\mathrm{a}_{\mathrm{F}} \div-\mathrm{l}_{\mathbb{1}}$
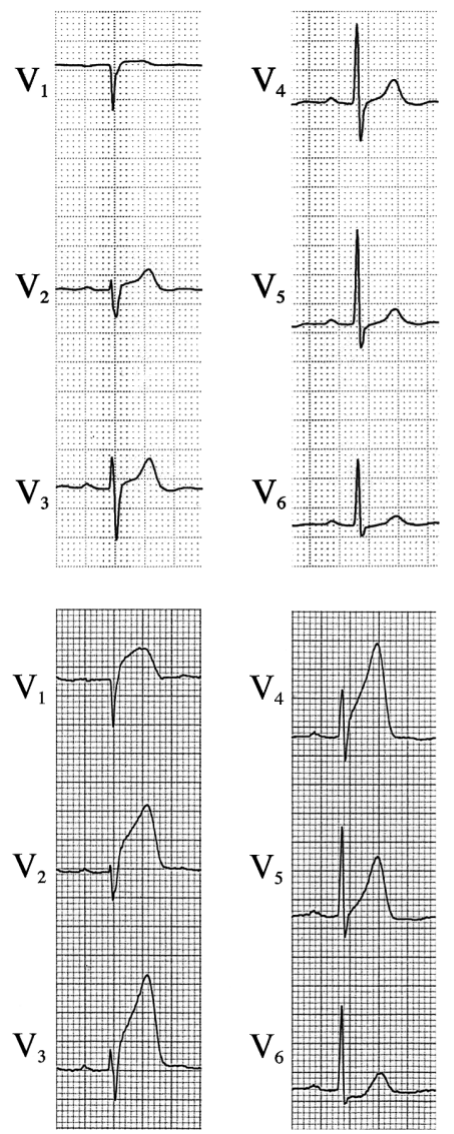

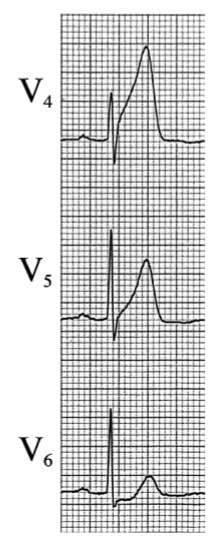

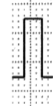

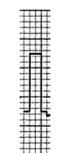

Fig 1. Electrocardiogram at the baseline showing normal findings (A). The electrocardiogram on admission showing ST elevation in the precordal leads (B).
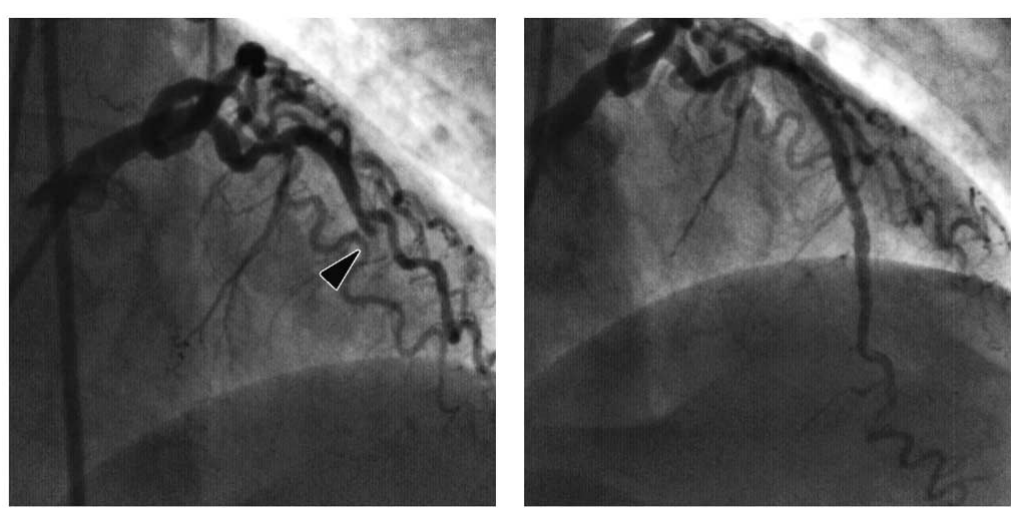

Fig 2. Coronary angiography (CAG) findings in the RAO cranial view. CAG revealed a 1-vessel coronary heart disease with total occlusion of the mid left anterior descending coronary artery (LAD) (Left). Two metal stents were implanted into the occluded lesion and recanalization of the LAD was achieved (Right).
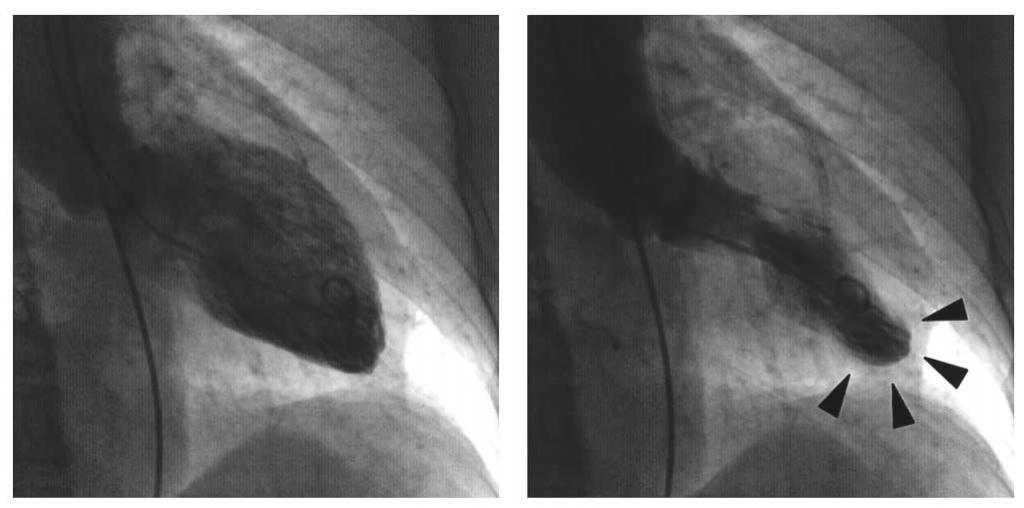

Fig 3. Left ventriculography findings in diastole (Left) and systole (Right). Apical akinesia (arrows) and posterobasal hypercontractility are evident. 


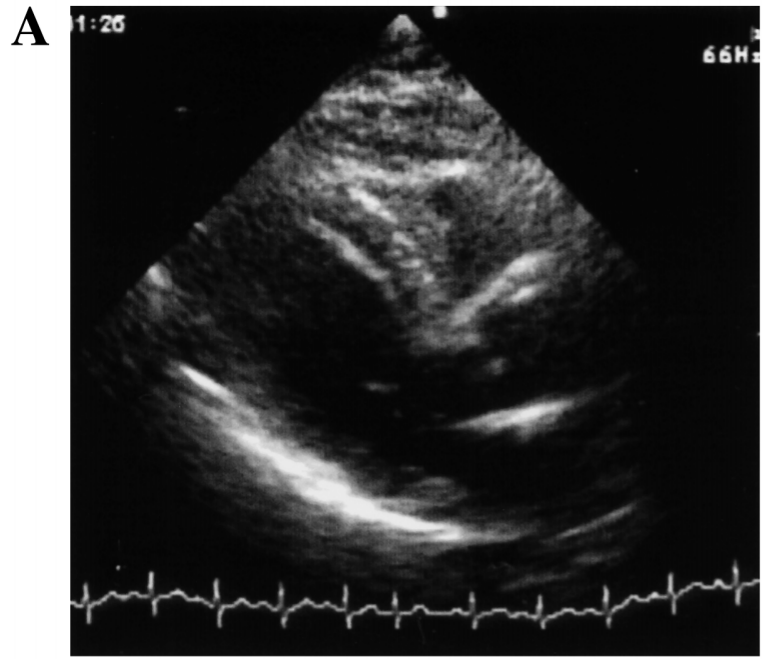

B

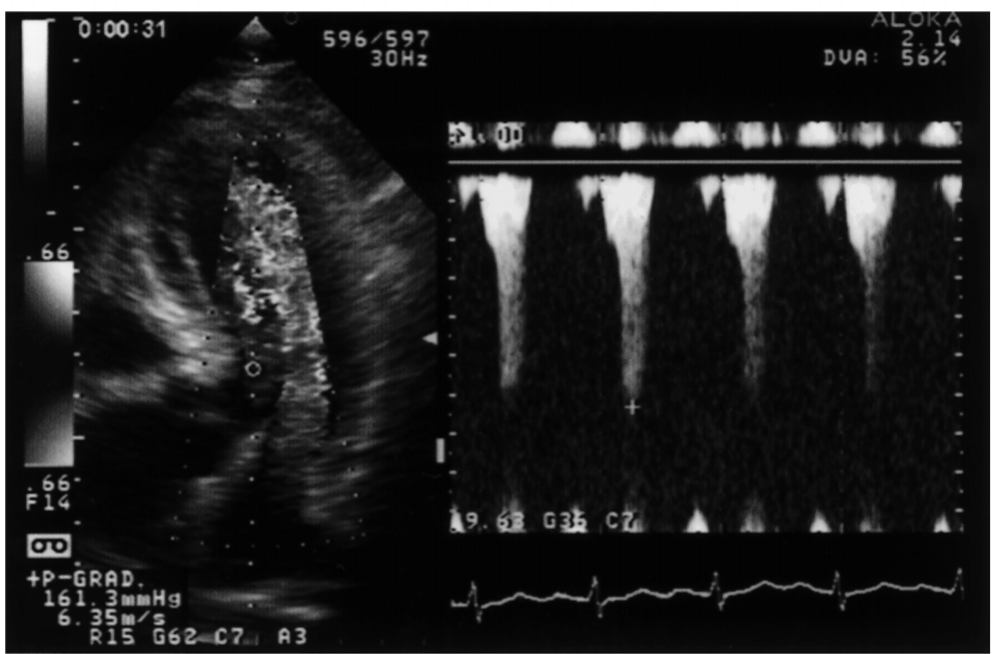

C

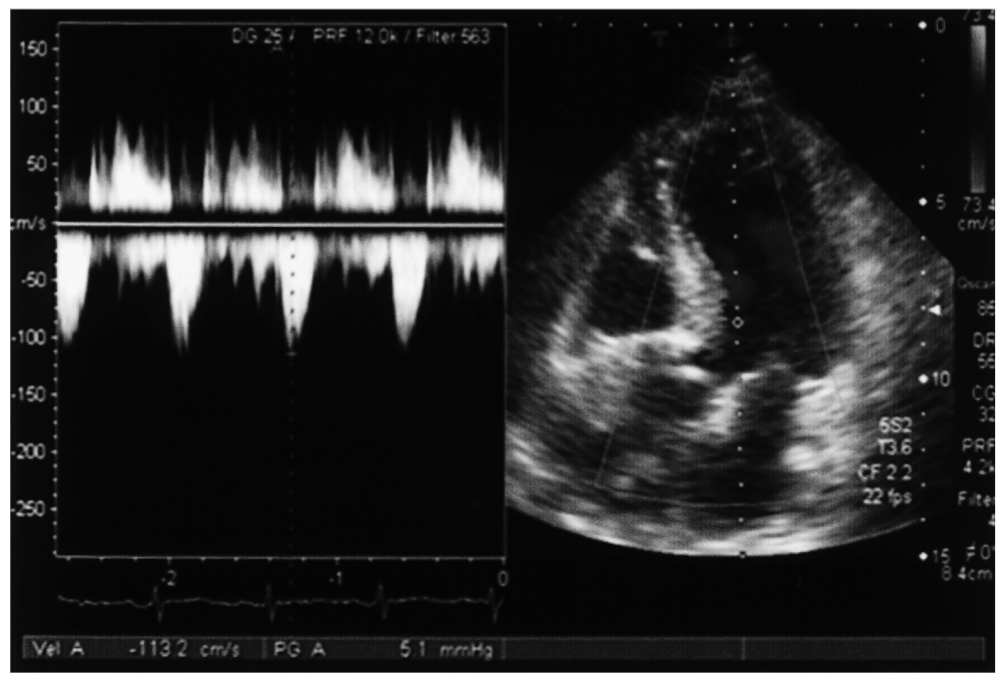

Fig 4. (A) Parasternal long-axis view of 2-dimensional echocardiography. The basal part of the interventricular septum bulged into the left ventricular outflow tract (LVOT). There were no indications of hypertrophic cardiomyopathy. (B) Spectral imaging of continuous wave Doppler of the LVOT from the apical position. The peak velocity value of $6.35 \mathrm{~m} / \mathrm{s}$ indicated a peak intraventricular gradient of $161 \mathrm{mmHg}$. (C) Spectral imaging of continuous wave Doppler of the LVOT from the apical position on day 2 after cilostazol treatment was terminated. The LVOT velocity was normal $(1.13 \mathrm{~m} / \mathrm{s})$. demonstrated apical hypokinesia and mild posterobasal and posterolateral hypercontractility. There was no pressure gradient between the apex and ascending aorta on pullback. After intravenous infusion of dobutamine, a $30 \mathrm{mmHg}$ pressure gradient was evoked between the apex and the LVOT (Fig 5). Hence, the patient was treated with atenolol $(25 \mathrm{mg} /$ day $)$ and she was discharged with no symptoms a few days later.
Informed consent was obtained before the provocation test with cilostazol and intravenous infusion test with dobutamine.

\section{Discussion}

In the present study case, the echocardiographic finding of the high velocity in LVOT revealed that the intraventricu- 


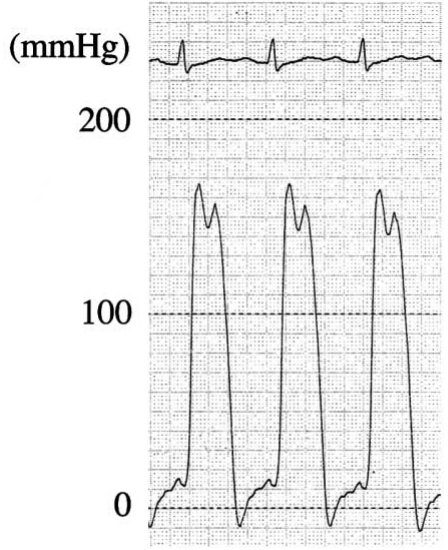

LV apex

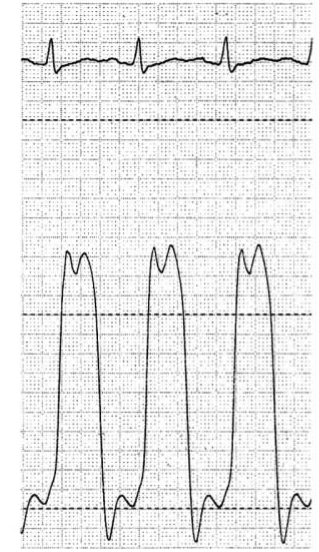

LVOT

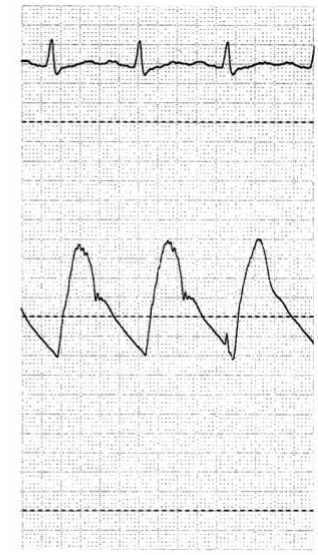

Ao
Fig 5. Findings of left ventricular (LV) and aortic (Ao) pressure after dobutamine infusion. Dobutamine infusion provoked a $30 \mathrm{mmHg}$ pressure gradient between the apex and the left ventricular outflow tract (LVOT). lar obstruction had caused the new systolic murmur. The degree of intraventricular obstruction was considered to change during the time-course of AMI and as a result of the use of cilostazol. However, the question remains: Why did the obvious intraventricular obstruction occur and disappear in our case with no findings of hypertrophic cardiomyopathy or severe concentric left ventricular hypertrophy, which are typically associated with dynamic intraventricular obstruction?8,9

First of all, there was the potential that this was caused by an abnormality of the left ventricular wall motion because of AMI. Echocardiography and LVG demonstrated apical akinesis with compensatory hyperdynamic contraction of the residual normally perfused basal segments. The development of compensatory hyperkinesis of the non-infarcted myocardium during acute ischemia has been described previously 10 This compensatory hyperkinesis during acute AMI narrows the systolic LVOT, resulting in acceleration of blood flow through the LVOT. In the sub-acute phase, this compensatory hyperkinesis disappeared, suggesting that the degree of the intraventricular obstruction weakened.

Second, the use of cilostazol was related to the occurrence of intraventricular obstruction. Cilostazol is a potent cyclic nucleotide phosphodiesterase type 3 (PDE3) inhibitor that has been approved for the treatment of peripheral artery disease, because of its vasodilatory and platelet activation inhibitory activities $!^{11}$ Through this PDE3 inhibitory activity, cilostazol also exerts positive cardiac inotropic effects. Armstrong et al reported 2 cases with AMI and transient intraventricular obstruction following the administration of intravenous inotropic drugs! Both cases were resolved by discontinuing administration of the intravenous inotropic drugs. In our case, intraventricular obstruction occurred with increased heart rate after administration of cilostazol. Moreover, the intraventricular obstruction disappeared after the withdrawal of cilostazol and the heart rate decreased. Inotropic agents including cilostazol must be considered as having the potential to deteriorate intraventricular obstruction as a complication with AMI.

Finally, the sigmoid-shaped ventricular septum was revealed by echocardiography in our case. Sigmoid septum is usually considered as a normal part of the aging process that is of little clinical importance, but several cases associated with outflow obstruction have been reported ${ }^{12-15}$ During the subacute phase of AMI in which there was a disappear- ance of the systolic murmur, provocation with dobutamine caused a $30 \mathrm{mmHg}$ pressure gradient between the apex and LVOT in this case. This phenomenon might be caused by the sigmoid septum as previously described 16 The sigmoidshaped ventricular septum is likely to be related to the intraventricular obstruction.

It is unknown which of the mechanisms were independent factors in our case. No intraventricular obstruction was evident during the emergent cardiac catheterization on the admission day. The intraventricular obstruction persisted only in the acute-term while cilostazol was administered. However, the re-administration of cilostazol and exercise during the subacute term did not provoke the intraventricular obstruction. Based on these facts, the intraventricular obstruction was considered to occur as a result of the coexistence of 3 factors, and the administration of cilostazol during the acute term might play an important role in the occurrence of intraventricular obstruction.

Dynamic intraventricular obstruction is a potentially reversible complication of AMI, if it is immediately diagnosed and treated appropriately. However, there have been some reports of patients with transient intraventricular obstruction during AMI who experienced myocardial rupture and subsequently died as a result 3,6 Dynamic intraventricular obstruction is initially diagnosed with the detection of a new ejection systolic murmur, as in this case. Mitral regurgitation and ventricular septal perforation are well-recognized complications of AMI with a new systolic murmur. To diagnose intraventricular obstruction, echocardiography can be extremely helpful in excluding other etiologies of systolic murmur, such as mitral regurgitation and ventricular septal perforation. Prompt diagnosis using echocardiography and early therapy are necessary to avoid a fatal outcome.

\section{References}

1. Armstrong WF, Marcovitz PA. Dynamic left ventricular outflow tract obstruction as a complication of acute myocardial infarction. Am Heart J 1996; 131: 827-830.

2. Joffe II, Riley MF, Katz SE, Ginsburg GS, Douglas PS. Acquired dynamic left ventricular outflow tract obstruction complicating acute anterior myocardial infarction: Serial echocardiographic and clinical evaluation. J Am Soc Echocardiogr 1997; 10: 717-721.

3. Bartunek J, Vanderheyden M, de Bruyne B. Dynamic left ventricular outflow tract obstruction after anterior myocardial infarction: A potential mechanism of myocardial rupture. Eur Heart J 1995; 16: $1439-1442$. 
4. Haley JH, Sinak LJ, Tajik AJ, Ommen SR, Oh JK. Dynamic left ventricular outflow tract obstruction in acute coronary syndromes: An important cause of new systolic murmur and cardiogenic shock. Mayo Clin Proc 1999; 74: 901-906.

5. San Roman Sanchez D, Medina O, Jimenez F, Rodriguez JC, Nieto V. Dynamic intraventricular obstruction in acute myocardial infarction. Echocardiography 2001; 18: 515-518.

6. Hrovatin E, Piazza R, Pavan D, Mimo R, Macor F, Dall'Aglio V, et al. Dynamic left ventricular outflow tract obstruction in the setting of acute anterior myocardial infarction: A serious and potentially fatal complication? Echocardiography 2002; 19: 449-455.

7. Safi AM, Rachko M, Kwan T, Tang A, Stein RA. Dynamic left ventricular outflow obstruction: A reversible mechanical complication of acute myocardial infarction. Angiology 2002; 53: 721-726.

8. Wigle ED, Rakowski H, Kimball BP, Williams WG. Hypertrophic cardiomyopathy: Clinical spectrum and treatment. Circulation 1995; 92: $1680-1692$.

9. Harrison MR, Grigsby CG, Souther SK, Smith MD, DeMaria AN Midventricular obstruction associated with chronic systemic hypertension and severe left ventricular hypertrophy. Am J Cardiol 1991; 68: $761-765$.

10. Grines CL, Topol EJ, Califf RM, Stack RS, George BS, Kereiakes D, et al. Prognostic implications and predictors of enhanced regional wall motion of the noninfarct zone after thrombolysis and angio- plasty therapy of acute myocardial infarction. Circulation 1989; 80: $245-253$.

11. Cone J, Wang S, Tandon N, Fong M, Sun B, Sakurai K, et al. Comparison of the effects of cilostazol and milrinone on intracellular cAMP levels and cellular function in platelets and cardiac cells. $J$ Cardiovasc Pharmacol 1999; 34: 497-504.

12. Dalldorf FG, Willis PW IV. Angled aorta ('sigmoid septum') as a cause of hypertrophic subaortic stenosis. Hum Pathol 1985; 16: $457-462$.

13. Iida K, Sugishita Y, Ajisaka R, Matsumoto R, Higuchi Y, Tomizawa $\mathrm{T}$, et al. Sigmoid septum causing left ventricular outflow tract obstruction: A case report. J Cardiogr 1986; 16: 237-247.

14. Yokoya T, Moromikawa J, Uehara K, Nakada K, Kuba M, Zaha M. Sigmoid shaped ventricular septum causing hemodynamic deterioration during lumbar anesthesia: A case report. J Cardiol 2002; 40: 167-172 (in Japanese with an English abstract).

15. Konishi C, Shiraishi J, Muraguchi N, Ohtsuki K, Inoue M, Tatsumi $\mathrm{T}$, et al. Benefical effect of cibenzoline on left ventricular pressure gradient with sigmoid septum. Circ J 2004; 68: 968-971.

16. Sonoda M, Takenaka K, Watanabe F, Igarashi T, Mashita M, Aoki T, et al. Dobutamine stress causes left ventricular outflow tract obstruction. J Cardiol 1996; 27(Suppl 2): 45-48 (in Japanese with an English abstract). 